\title{
Sistema de videocolaboração para o museu interativo do TCE-PB baseado no modelo DA
}

\author{
Amanda Azevedo \\ Design Audiovisual/LAVID/UFPB \\ João Pessoa, Brasil \\ amanda.azevedo@lavid.ufpb.br \\ Raoni Kulesza \\ LAVID/CI/UFPB \\ João Pessoa, Brasil \\ raoni@lavid.ufpb.br
}

\author{
Sandro Gonçalves \\ Design Audiovisual/LAVID/UFPB \\ João Pessoa, Brasil \\ sandro.goncalves@lavid.ufpb.br
}

\author{
Fábia Carolino \\ TCE-PB \\ João Pessoa, Brasil \\ fluna@tce.pb.gov.br
}

\author{
Valdecir Becker \\ Design Audiovisual/LAVID/CI/UFPB \\ João Pessoa, Brasil \\ valdecir@ci.ufpb.br
Agnaldo Macedo Filho
TCE-PB
João Pessoa, Brasil
amfilho@tce.pb.gov.br

\begin{abstract}
This work describes a system focused on interactive audiovisual experiences developed for the State of Paraíba Court of Auditors (TCE-PB). The system includes professional and amateur productions to enable social control using multimedia technologies. As a result, the video collaboration solution, based on the Audiovisual Design model, is presented.
\end{abstract}

\section{KEYWORDS}

Audiovisual Design, Social Control, Fruition.

\section{INTRODUÇÃO}

O Museu Interativo do Tribunal de Contas do Estado da Paraíba TCE-PB é um projeto com o objetivo de conscientizar a população sobre o controle social, que usa tecnologias da informação e comunicação. Para tanto, a produção de conteúdos audiovisuais, dispostos em ambientes multimídias, compõe uma estratégia de disseminação de uma cultura de fiscalização. Esse projeto é uma parceria do Grupo de Pesquisa Design Audiovisual com o Laboratório de Aplicações em Vídeo Digital (LAVID) e o Tribunal de Contas do Estado da Paraíba (TCE-PB). O Projeto é composto por aplicativos, games, ambientes de realidade aumentada e conteúdos audiovisuais. Este artigo descreve os sistemas de fruição de produções profissionais e colaborativas do componente audiovisual do Museu, chamado Cine-TCE [1]. O escopo deste módulo inclui as produções audiovisuais interativas do projeto sendo apresentados os sistemas de Gerenciador de Conteúdos no

In: V Workshop "O Futuro da Videocolaboração" (WCT-Video 2018), Salvador, Brasil. Anais do XXIV Simpósio Brasileiro de Sistemas Multimídia e Web: Workshops e Pôsteres. Porto Alegre: Sociedade Brasileira de Computação, 2018. (c) 2018 SBC - Sociedade Brasileira de Computação. ISBN: 978-85-7669-435-9. portal web e três ambientes de fruição e visualização: o Mapa Colaborativo no modo web, que exibe todos os conteúdos enviados, a Mesa Interativa em painéis sensíveis ao toque, encontrada no Museu, e um sistema de Apoio a Palestras, onde os conteúdos disponíveis podem ser incluídos em apresentações no Auditório do TCE-PB. São contempladas duas formas de entrada de conteúdos: vídeos institucionais, produzidos pelo LAVID, que retrata a atuação do TCE-PB; e vídeos de curta duração produzidos por estudantes da rede pública do estado da Paraíba, que mostra as dificuldades de execução em projetos públicos e sugere soluções para tais problemas.

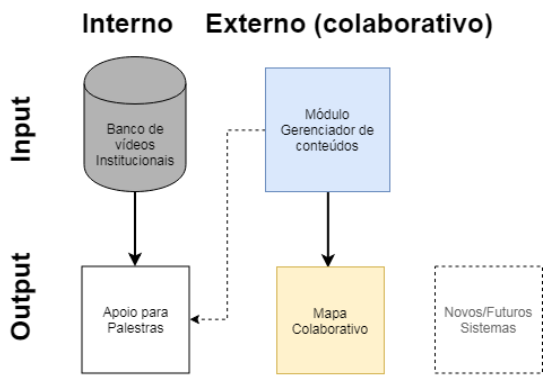

Figura 1. Relação entre conteúdos e formas de visualização.

Conforme descrito na figura 1 , cada entrada de conteúdo tem uma abordagem de visualização direta. Considera-se também nesta proposta a possibilidade de utilização de dados do Módulo de Gerenciamento de Conteúdos para desenvolver funções ou atividades dentro do Sistema de Apoio a Palestras bem como futuros sistemas.

O Museu busca tornar a visitação contínua potencializando o programa já existente TCE Escola e Cidadania, no qual os estudantes tem acesso ao acervo do Memorial e a palestras no auditório do TCE-PB, sobre a função na auditoria das contas públicas, combate a corrupção e controle social. O projeto foca na rede estadual de ensino, tanto na produção dos conteúdos, quanto 
nas visitas ao Museu. Para tanto, pretende-se usar os recursos do Museu para conscientizar os estudantes e professores sobre a importância do controle social. Conteúdos como cidadania, políticas públicas e convivência em sociedade, que já fazem parte do dia a dia dos estudantes, servirão de base para a disseminação da cultura de fiscalização.

\section{MATERIAIS E MÉTODOS}

Para desenvolver os cenários de fruição, o Design Audiovisual foi utilizado como modelo teórico e método. O Design Audiovisual é um modelo conceitual e um processo metodológico para a criação e avaliação de conteúdos audiovisuais, mediados por interfaces digitais, integrando de forma interdisciplinar os conceitos e processos dos estudos de mídia e Interação Humano Computador. Aplicações e análises deste modelo foram realizadas em estudos anteriores de fruição audiovisual [5] [6] [7] [8] [9]. O modelo descreve quatro papéis que os indivíduos assumem ao consumir conteúdos audiovisuais: Audiência, Sintetizador, Modificador e Produtor. O papel da Audiência representa o comportamento "passivo" do indivíduo diante da fruição, como a visualização dos conteúdos. As ações do Sintetizador são: compilar, classificar, comentar, recomendar e compartilhar o conteúdo, construindo, dessa forma, uma "identidade" digital, um perfil encenado em uma rede social. O Modificador domina softwares para manipular e recriar conteúdos, ampliando a noção de engajamento para a de apropriação. O papel do Produtor são os responsáveis pelo design e criação dos conteúdos, seja de forma autônoma ou em grupos de mídia. Cada papel tem seus níveis elevados, descritos como Players, quando o indivíduo se envolve e usa os recursos disponíveis da interface para fruir um conteúdo audiovisual. As mudanças de papéis ocorrem quando há um aumento do engajamento e da interação do indivíduo com os sistemas, quando há a percepção da qualidade do conteúdo junto a utilidade do software.

Os sistemas apresentados promove uma experiência direcionada à facilitação da visualização, filtragem e personalização de conteúdos para envolver o indivíduo nos níveis de engajamento apresentado nos papéis de Audiência.As etapas apresentadas explanam desde a idealização do projeto até a implementação das soluções. A criação da proposta, as possibilidades de comunicação e interação, e a realização da pesquisa de usuário com alunos das escolas públicas seguiram as Linhas de Design do modelo de Design Audiovisual. Finalmente, o projeto foi formalizado e implementado usando métodos ágeis da engenharia de software.

\section{CENÁRIOS DE TESTES}

Nos tópicos seguintes descreveremos como cada sistema supre as demandas do Museu Interativo do TCE-PB, sendo elaborado por etapas, seguido dos cenários de testes em telas de navegação.

Etapa 1: Delimitamos um público específico para imergir na proposta do projeto. Alunos, professores e palestrantes foram os indivíduos guiadores para o desenvolvimento dos sistemas e estratégias de produção de conteúdos audiovisuais.

Etapa 2: A produção de um portal web do projeto que inclui um Gerenciador de Conteúdos, servindo como ferramenta para entrada dos dados através do envio dos vídeos, e concentra os conteúdos que são acessados pelos demais sistemas. No portal o professor realiza o cadastro e interage com as funcionalidades de gerenciamento referentes aos envios, seleções e alterações dos vídeos dos alunos.
Etapa 3: O sistema do Mapa Colaborativo corresponde à visualização dos conteúdos, sendo alimentado pelos vídeos enviados através do Gerenciador. $\mathrm{O}$ sistema tem uma página à parte do portal, contendo o mapa da Paraíba, em que cada município é um ponto de acesso aos respectivos conteúdos dos alunos.

Etapa 4: O segundo sistema de visualização é uma representação pelo mesmo Mapa Colaborativo web, mas acessível por uma Mesa Interativa touchscreen e um projetor de curta distância, dispostos dentro do Museu. Esse sistema permite, além da visualização, a criação de playlists para exibição.

Etapa 5: O terceiro sistema é o de personalização de palestras, dedicados exclusivamente aos funcionários do TCE-PB que ministram essas apresentações durante a visita das escolas no auditório. O sistema oferece ao palestrante formas de mapear e escolher os vídeos compondo uma apresentação mais atrativa para os visitantes.

\subsection{Inputs de Interação}

Diante destas aplicações descreveremos detalhadamente como ocorrerá o funcionamento técnico, a interação com as interfaces e a relação com os conteúdos audiovisuais do Museu com base nos inputs de fruição dos sistemas.

\subsubsection{Gerenciador de Conteúdos}

Os casos de uso neste sistema são a realização do cadastro pelo professor, que preenche um formulário com dados pessoais, fornecendo também sua matrícula da rede de ensino para que o cadastro seja autenticado no sistema.

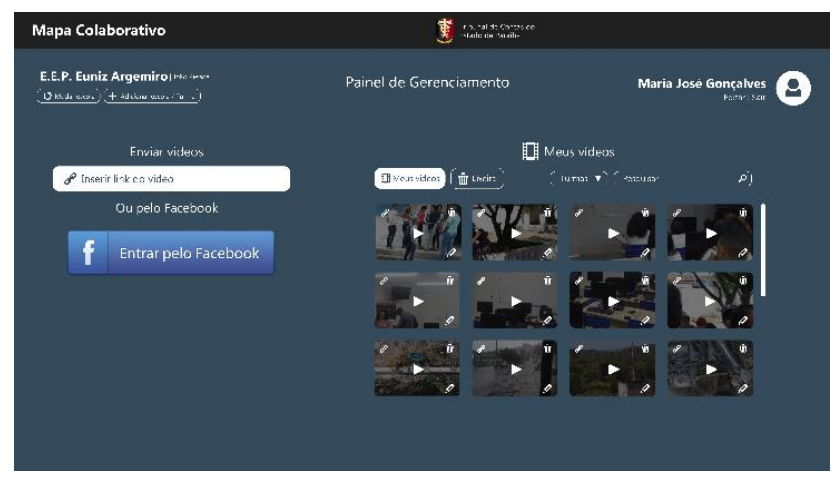

Figura 2: Parte da interface do sistema de Gerenciamento de Conteúdos

Realizado o cadastro e o login, o professor é guiado à interface principal do Gerenciador, onde pode adicionar os vídeos via desktop, autorizar os conteúdos dos alunos usando as postagens dos perfis do Facebook captados por API Graph, ativar as permissões para a publicação do conteúdo na plataforma ou, ainda, inserir o link do vídeo para que os conteúdos sejam direcionados ao Mapa Colaborativo. Os requisitos funcionais do sistema são: login, upload, remoção e edição de vídeos, filtro por escolas e turmas participantes.

\subsubsection{Mapa Colaborativo}

Os conteúdos enviados pelo Gerenciador de Conteúdos servirá como input para o Mapa Colaborativo. A visualização no mapa 
pode ser acessada no modo web e na Mesa Interativa disposta no Museu.

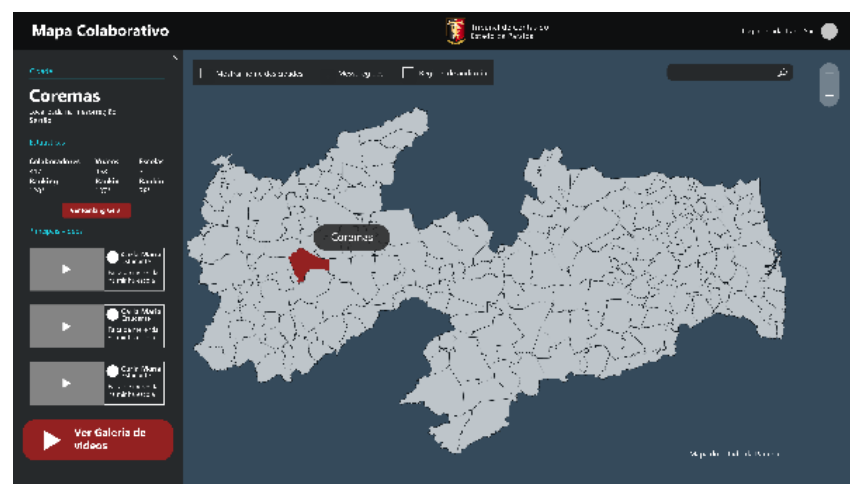

Figura 3: Parte da interface do sistema do Mapa Colaborativo.

Os requisitos para o Mapa Colaborativo no modo web são: o acesso pelo local de envio, regiões de auditoria do TCE-PB e na galeria de vídeos pela busca das escolas e acesso ao player de vídeo.

\subsection{Outputs de Fruição}

As saídas dos sistemas do TCE-PB corresponde a personalização de vídeos, mesclando autorias dos vídeos profissionais e dos colaborativos, que promove experiências com os conteúdos de forma inédita nas visitais ao Museu.

\subsubsection{Mesa Interativa}

Para a interação na Mesa são acrescentados os requisitos de criação de playlists de exibição. Os casos de uso para a Mesa Interativa são as sessões de exibição no Museu que permite a visualização de forma coletiva. Como requisitos desejáveis será necessário inserir uma página de login para o professor, que guiará as sessões junto aos alunos no Museu, possa elaborar uma personalização de playlists com os vídeos, que poderão gerar ao final da sessão sua propagação através do compartilhamento nas redes sociais usando uma URL ou QR Code.

\subsubsection{Apoio a Palestras}

O sistema de Apoio a Palestras é de uso dos palestrantes para criar playlists de vídeos institucionais e colaborativos, de acordo com o objetivo da apresentação. Esse sistema permite a escolha de trechos ou dos vídeos completos. Os requisitos deste sistema são a criação de apresentações, mapeamento dos conteúdos, ajustes, disponibilização de apresentações já realizadas que ficam registradas no sistema e player de vídeos acessível por aplicativo mobile, para controle da apresentação. Os requisitos desejáveis deste sistema será o acesso a URL ou QR Code da apresentação personalizada do palestrante para que os alunos possam compartilhar em suas redes sociais, que ao identificar suas autorias torna-se um fator atrativo para estimular a propagação.

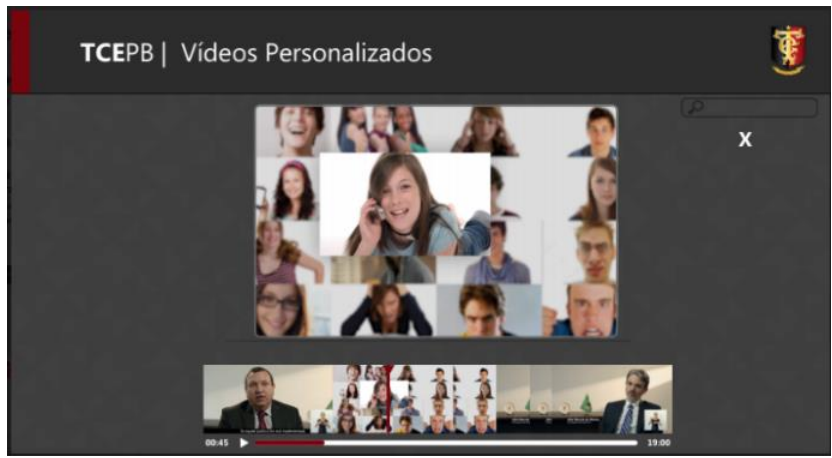

Figura 4. Parte da interface do sistema de Apoio a Palestras.

Todo o sistema está instalado localmente, com conexão entre dispositivos feita por rede local. A realização de testes focou nos requisitos de login e envio de vídeos como etapas iniciais de implementação para a primeira versão da aplicação.

\section{CENÁRIOS DE PRODUÇÃO E CONSUMO PELAS LINHAS DE DESIGN AUDIOVISUAL}

O uso integrado do conteúdo e interface em cenários multimídias resulta em experiências de fruição cada vez mais complexas. Conforme apresentado no estudo sobre arquitetura de design colaborativo [6], o indivíduo ao explorar o conteúdo se relaciona com a interface em momentos passivos de visualização e momentos ativos de interação e propagação. Os papéis de Audiência do Design Audiovisual são frutos de ações baseadas nas quatro linhas conceituais de Design: Identidade, Motivação, Experiência e Conteúdo que visam uma experiência focada nos fatores humanos do indivíduo, gerando novas formas de transmissão e recepção de conteúdos, diferente como encontrado no modelo Broadcast [5].

Demonstramos, portanto como ocorre o percurso de interação que conduz as experiências de fruição entre indivíduo e sistemas, por meio das demandas de produção e consumo.

\subsection{Linha do Conteúdo}

A linha do conteúdo é definida pelas práticas de produção audiovisual, em que o estudante que produz conteúdo assume o papel do Produtor, de criador da obra, desde a concepção estética à criação do conteúdo.

\subsection{Linha da Experiência}

Esta linha foca no envolvimento, nas curiosidades e desafios que o indivíduo realiza para fruir o conteúdo, elevando suas ações a níveis extremos, que corresponde a características de mais de um papel e emerge o Player. Cada percurso de interação promove experiências diferenciadas. $\mathrm{O}$ uso do Mapa Colaborativo proporciona o indivíduo a visualizar o conteúdo, configurando-se Audiência, e possibilita alterar um momento passivo para ativo ao se relacionar com suas funcionalidades como: selecionar vídeos usando as ferramentas de busca tornando-se Audiência-Player, apoiar e comentar os vídeos gerando competições, atuando como Sintetizador-Player.

\subsection{Linha da Identidade}

As ações nesta linha são geradas por preferências e personalizações que o indivíduo realiza no processo de fruição. A 
experiência no Museu através da Mesa Interativa gera alternância entre as ações de Audiência (visualização) para Modificador (novas possibilidades de conteúdo), que ao elaborar playlists organizadas em categorias de temas, escolas ou vídeos mais apoiados, alteram o sentido inicial e seus contextos, gerando um novo produto. No caso de uso do sistema de Apoio a Palestras os papéis de Audiência e Sintetizador estão mais evidentes entre os estudantes, que ao assistir a palestra atua como Audiência e ao receber o vídeo personalizado compartilhando nas redes sociais torna-se um Sintetizador, incorporando suas opiniõs, críticas e configurando um "perfil” de conteúdo a ser exposto nas redes sociais.

\subsection{Linha da Motivação}

No processo de fruição o indivíduo possui uma intenção, que busca atender as suas necessidades intrínsecas e extrínsecas. Identificamos os principais casos de uso focados na motivação. O professor recebe os conteúdos disponibilizados pelos alunos, submetendo ao Gerenciador de Conteúdos, tornando-se assim Sintetizador. A mediação é focada na motivação para a produção destinada aos os alunos, bem como selecionar os

conteúdos que devem representar a escola, sendo indispensável sua participação.

O palestrante no momento que realiza a seleção dos vídeos para gerar uma nova apresentação atua como Modificador, no qual busca despertar o engajamento da Audiência para o que pretende comunicar na palestra.

O foco das linhas de Design é direcionar a experiência para a mudança dos papéis, que não necessariamente são lineares, sendo baseado em conjuntos, tornando-se dinâmicos. A experiência de fruição pode ocorrer em dois ou mais papéis simultaneamente: $o$ indivíduo que assiste uma obra (Audiência), participa de discussões nas redes sociais (Sintetizador) e interfere no conteúdo ao remixar a produção inserindo suas impressões e gerando novos sentidos (Modificador ou Produtor).

\section{RESULTADOS}

Os sistemas do museu tem as linhas de Design como modelagem do produto, fazendo uso de Affordances que foram pensadas para as ações de produção, personalização e propagação de conteúdo audiovisual em um ambiente midiático, de acordo com a proposta do museu. As Media Affordances [7] tem como objetivo guiar o indivíduo através da correta percepção associadas ao seu contexto no ambiente de fruição que envolve percepções físicas (affordances que podem ser ativamente manipuladas, com usos potenciais facilmente perceptíveis, compostas por tecnologias usadas para fruição de conteúdos), gráficas (affordances presentes em interfaces gráficas interacionais implementadas por software e responsáveis por parte da mediação entre indivíduos e conteúdo) e simbólicas (affordances presentes na narrativa ou inseridas no conteúdo audiovisual, onde as affordances físicas são mais sutis e as relações cognitivas e sensoriais dependem não apenas de tecnologia e interfaces, mas também da compreensão de elementos narrativos). Com base nas Media Affordances são estabelecidos Gatilhos de Interação (Interaction Triggers - IT), divididos em Ação (Triggers of Action - ToA) e Inércia (Triggers of Inertia - ToI) que possibilitam ao indivíduo desempenhar a função dos papéis de Audiência. Com base nesses parâmetros serão avaliados as produções dos alunos e a os níveis de engajamento identificando se atinge ou não os objetivos necessários para cada papel, que desempenham a videocolaboração dentro do sistema.

\section{CONSIDERAÇÕES FINAIS}

Essas apropriações dos conteúdos e integração no uso dos sistemas apontam para novas perspectivas de autorias, personalização e modificação em sistemas colaborativos, gerando produtos multimídias inovadores. Como perspectiva futura deste projeto aponta-se as novas opções de fruição descritos como requisitos desejáveis. Esse tipo de propagação permitirá que se atinja um maior número de pessoas através das estratégias apresentadas. Diante deste compartilhamento surgião novas implicações do conhecimento acerca do Museu e, consequentemente, no uso dos demais sistemas.

\section{AGRADECIMENTOS}

Os autores gostariam de agradecer a Marianna Teixeira, Jaqueline Donin e Thiago Vasconcelos, cujas pesquisas foram fundamentais para a viabilização da presente proposta.

\section{REFERÊNCIAS}

[1] CHAUDHRY, A. N. TOSCANO, R. M. N. AZEVEDO, A. S. BECKER, V. Pesquisa documental e propostas de identidade visual e de interação. Módulo WP7 Cine do projeto Espaço Interativo do TCE, abril, 2018.

[2] CHAUDHRY, A. N. TOSCANO, R. M. N. AZEVEDO, A. S. SOUZA, H. DONIN. J. BECKER, V. Pesquisa documental, propostas e protótipos para vídeo e interação. Módulo WP7 Cine do projeto Espaço Interativo do TCE, maio, 2018.

[3] CHAUDHRY, A. N. TOSCANO, R. M. N. AZEVEDO, A. S. SOUZA, H. BECKER, V. Pesquisa e produção de conteúdo. Módulo WP7 Cine do projeto Espaço Interativo do TCE, junho, 2018

[4] TOSCANO, R. M. N. AZEVEDO, A. S. GONÇALVES, S. DONIN, J. TEIXEIRA, M VASCONCELOS, T. CHAUDHRY, A. N. BECKER, V. Produção de vídeos institucionais, visualização e interação com o conteúdo. Módulo WP7 Cine do projeto Espaço Interativo do TCE, julho, 2018

[5] BECKER, V. GAMBARO, D. RAMOS, T. Audiovisual Design and the Convergence Between HCI and Audience Studies. Springer International Publishing AG 2017.

[6] TOSCANO, R. M. N. BECKER, V. SILVEIRA, L. COUTINHO, S. BURGOS, L. Arquitetura de design colaborativo para imersão temporal e espacial em vídeos de altíssimas resoluções e HFR. O futuro da vídeocolaboração: perspectivas. In S. B. de Computação (Ed.) Gramado, RS, 2017.

[7] BECKER, V. GAMBARO, D. RAMOS, TOSCANO, R.M.N Audiovisual Design: Introducing "Media Affordances" as a Relevant Concept for the Development of a New Communication Model. Applications and Usability of Interactive Television. 6th Iberoamerican Conference, jAUTI. Aveiro, Portugal, October, 2017.

[8] BECKER, V., GAMBARO, D., RAMOS, T. S., \& BEZERRA, E. P. (2018). Design Audiovisual: a interseção dos estudos de audiência com a Interação Humano Computador. Conexão-Comunicação e Cultura, 17(33).

[9] GAMBARO, D., BECKER, V., RAMOS, T. S., \& TOSCANO, R. (2018). The Development of Individuals' Competencies as a Meaningful Process of the Audiovisual Design Methodology. In International Conference on Human-Computer Interaction (pp. 68-81). Springer, Cham. 